\title{
Pandrug-resistant Infections
}

\author{
Novy Gupte ${ }^{1}$, Sapna Pradhan ${ }^{2}$
}

\begin{abstract}
Background: Increasing antibiotic resistance, usually from irrational pharmacotherapy, poses a grave challenge to clinicians in managing multidrug-resistance infections.

Aim and objective: To focus attention on the rising incidence of pandrug-resistant infections, related issues and concerns, and their containment. Materials and methods: The short communication is prompted by the recently reported in vitro sensitivity of gram-negative bacteria to a combination of ceftriaxone + sulbactam + EDTA. Appropriate observations from literature are cited to complement the outcome of the said study. Results: Pandrug resistance (PDR) is defined as a non-susceptibility of the bacteria to all antimicrobial agents in all antimicrobial categories. This is not true that it is restricted to only gram-negative bacteria. Staphylococcal aureus, a gram-positive bacteria, may too develop such resistance. The in vitro sensitivity observations do not necessarily get reflected in actual clinical effectiveness and efficacy. Therapy is a herculean task. Judicious use of antibiotics and strict infection control measures, preferably as a part of an antibiotic stewardship program, are mandatory to reduce the prevalence of PDR, nay the drug resistance as such.

Conclusion: Pandrug resistance, meaning resistance to all classes of antibiotics, can develop not only in the case of gram-negative bacteria but also gram-positive bacteria, like S. aureus. It is best prevented rather than treated.

Clinical significance: Implementation of the antibiotic stewardship program, mainly comprising rational antibiotic therapy, has the potentials to go a long way in safeguarding against PDR, nay antibiotic resistance as such.

Keywords: Acinetobacter, Antimicrobial resistance, Antimicrobial stewardship, Enterococcus, Extensive-drug resistance, Klebsiella pneumoniae, Pandrug resistance, Proteus, Pseudomonas aeruginosa, Staphylococcus aureus.

Journal of Medical Academics (2021): 10.5005/jp-journals-10070-0065
\end{abstract}

\section{INTRODUCTION}

The research paper by Yadav et al. ${ }^{1}$ reporting that pan-resistant infections (PDR) by Enterobacteriaceae and Proteus are susceptible to the combination of ceftriaxone + sulbactam + EDTA, makes interesting reading.

In this context, I wish to share the following observations for the benefit of your readers.

First, undoubtedly further in vitro studies with special reference to minimum inhibitory concentration (MIC) and minimum bactericidal concentration (MBC) in different centers are warranted to confirm the findings of the aforesaid study. Moreover, in vivo studies involving actual patients in healthcare centers too are needed. This is a fact that the in vitro sensitivity observations do not necessarily get reflected in actual clinical effectiveness and efficacy. Mercifully, in the recent past, integration of the pharmacodynamic and pharmacokinetic indices has considerably enhanced the correlation between in vitro susceptibility testing and in vivo clinical effectiveness. As a result, now more realistic breakpoints can be allowed.

Second, pandrug resistance (PDR) to gram-negative bacteria Pseudomonas aeruginosa, Klebsiella pneumoniae, Proteus, Enterobacteriaceae, and Acinetobacter is on an increase globally. Nonetheless, this is not true that PDR is restricted to only gramnegative bacteria. Staphylococcus aureus, a gram-positive bacteria, may too develop PDR.

Third, PDR infections are a huge challenge in pharmacotherapeutics. Individuals having health issues or compromised immune systems are often at the highest risk for getting such an infection. The two most common types of bacteria that can become resistant to antibiotics are S. aureus and Enterococcus.

Fourth, there is considerable confusion in the literature about what really constitutes PDR, especially in relation to extensive
1,2Department of Pharmacology, Army College of Medical Sciences, Delhi Cantonment, New Delhi, India

Corresponding Author: Novy Gupte, Department of Pharmacology, Army College of Medical Sciences, Delhi Cantonment, New Delhi, India, Phone: +91 8178971105, e-mail: drnovyguptemd@gmail.com

How to cite this article: Gupte N, Pradhan S. Pandrug-resistant Infections. J Med Acad 2021;4(1):28-29.

Source of support: Nil

Conflict of interest: None

drug resistance (XDR). ${ }^{2}$ As clarified in Table $1, \mathrm{XDR}$ is resistance to all classes of antibiotics except 1 or 2 . On the other hand, PDR is resistant to all classes of antibiotics. In other words, there is a non-susceptibility of the bacteria to all antimicrobial agents in all antimicrobial categories. The term, PDR, has also been employed for pathogens that are specifically resistant to seven antimicrobial agents (cefepime, ceftazidime, imipenem, meropenem, piperacillintazobactam, ciprofloxacin, and levofloxacin).

While efforts at determining a combination of potential antimicrobials with beta-lactamase inhibitors are in progress, research endeavors need to be boosted for the development and introduction into clinical practice of new antimicrobial agents.

Table 1: Precise definition of three types of antibiotic resistance Multidrug-resistance (MDR): Resistance to $\geq 3$ major classes of antibiotics.

Extensive drug-resistance (XDR): Resistance to all classes of antibiotics except one or two.

Pandrug resistance (PDR): Resistance to all classes of antibiotics.

(O) The Author(s). 2021 Open Access This article is distributed under the terms of the Creative Commons Attribution 4.0 International License (https://creativecommons. org/licenses/by-nc/4.0/), which permits unrestricted use, distribution, and non-commercial reproduction in any medium, provided you give appropriate credit to the original author(s) and the source, provide a link to the Creative Commons license, and indicate if changes were made. The Creative Commons Public Domain Dedication waiver (http://creativecommons.org/publicdomain/zero/1.0/) applies to the data made available in this article, unless otherwise stated. 
Finally, prevention of PDR infections, a public health problem, ${ }^{3}$ needs a special emphasis. Judicious use of antibiotics and strict infection control measures are mandatory to reduce the prevalence of drug resistance. The proactive propagation of antimicrobial stewardship program ${ }^{4}$ in letter and spirit in healthcare facilities may prove a "game changer" in containing the malady.

\section{References}

1. Yadav S, Mangalesh S, Makkar A, et al. In vitro evaluation of a novel derivative combination antimicrobial ceftriaxone + sulbactam +
EDTA against panresistant gram-negative bacteria at a 1,000bedded tertiary care teaching hospital in northern India. J Med Acad 2020;3(1):11-13. DOI: 10.47008/ajm.2020.3.1.3.

2. Magiorakos AP, Srinivasan A, Carey RB, et al. Multidrug-resistant, extensively drug-resistant and pandrug-resistant bacteria. Clin Microb Infect 2011;8(3):268-281.

3. Gupte N. Antimicrobial resistance: time to fight it out. Pediat Infect Dis 2012;1(4):15-17.

4. Gupte N. Antimicrobial stewardship: the crying need of the time. EC Pharama Toxicol (London) 2020;8(8):1-2. 